OPEN ACCESS

Edited by:

Yongming Qiu,

Shanghai JiaoTong University, China

Reviewed by:

Bharat Guthikonda,

Louisiana State University Health

Shreveport, United States

Bo Deng,

Daping Hospital, China

*Correspondence:

Ling Chen

chen_ling301@163.com

Chuan Xu

xuchuan100@163.com

${ }^{t}$ These authors have contributed equally to this work and share first authorship

Specialty section: This article was submitted to Neuro-Oncology and Neurosurgical Oncology, a section of the journal

Frontiers in Oncology

Received: 02 September 2021 Accepted: 24 November 2021 Published: 17 December 2021

Citation:

Shi Y, Wu M, LiU Y, Hu L, Wu H, Xie L, LiU Z, Wu A,

Chen $L$ and $X u C$ (2021)

ITGA5 Predicts Dual-Drug

Resistance to Temozolomide and Bevacizumab in Glioma.

Front. Oncol. 11:769592. doi: 10.3389/fonc.2021.769592

\section{ITGA5 Predicts Dual-Drug Resistance to Temozolomide and Bevacizumab in Glioma}

\author{
Ying Shi ${ }^{1 \dagger}$, Mengwan $\mathrm{Wu}^{1,2 \dagger}$, Yuyang $\mathrm{Liu}^{3}$, Lanlin $\mathrm{Hu}^{1,2}$, Hong $\mathrm{Wu}^{1,2}$, Lei Xie ${ }^{1}$, \\ Zhiwei Liu ${ }^{4}$, Anhua $\mathrm{Wu}^{5}$, Ling Chen ${ }^{3 *}$ and Chuan $\mathrm{Xu}^{1,2^{*}}$ \\ 1 Integrative Cancer Center \& Cancer Clinical Research Center, Sichuan Cancer Hospital \& Institute, Sichuan Cancer Center, \\ School of Medicine, University of Electronic Science and Technology of China, Chengdu, China, ${ }^{2}$ Department of Radiation \\ Oncology, Sichuan Cancer Hospital, Chengdu, China, ${ }^{3}$ Chinese People's Liberation Army (PLA) Institute of Neurosurgery, \\ Chinese PLA General Hospital and PLA Medical College, Beijing, China, ${ }^{4}$ The Center for Advanced Semiconductor \& \\ Integrated Micro-System, University of Electronic Science and Technology of China, Chengdu, China, ${ }^{5}$ Department of \\ Neurosurgery, The First Affiliated Hospital of China Medical University, Shenyang, China
}

Aims: Anti-angiotherapy (Bevacizumab) is currently regarded as a promising option for glioma patients who are resistant to temozolomide (TMZ) treatment. But ongoing clinical research failed to meet therapeutic expectations. This study aimed to explore the pivotal genetic feature responsible for TMZ and Bevacizumab resistance in glioma patients.

Methods: We downloaded the transcriptomic and methylation data of glioma patients from The Cancer Genome Atlas (TCGA), Chinese Glioma Genome Atlas (CGGA), and Gene Expression Omnibus (GEO) databases and grouped these patients into resistant and non-resistant groups based on their clinical profiles. Differentially expressed genes and pathways were identified and exhibited with software in R platform. A TMZ-resistant cell line was constructed for validating the expression change of the candidate gene, ITGA5. An ITGA5-overexpressing cell line was also constructed to investigate its biological function using the CCK8 assay, Western blot, periodic acid-Schiff (PAS) staining, and transcriptional sequencing.

Results: Change of the cell morphology and polarity was closely associated with TMZ mono-resistance and TMZ/Bevacizumab dual resistance in glioma patients. The expression level of ITGA5 was effective in determining drug resistance and the outcome of glioma patients, which is regulated by methylation on two distinct sites. ITGA5 was augmented in TMZ-resistant glioma cells, while overexpressing ITGA5 altered the cell-promoted TMZ resistance through enhancing vascular mimicry (VM) formation correspondingly.

Conclusions: Both the epigenetic and transcriptional levels of ITGA5 are effective in predicting TMZ and Bevacizumab resistance, indicating that ITGA5 may serve as a predictor of the treatment outcomes of glioma patients.

Keywords: glioma, TMZ resistant, Bevacizumab resistant, ITGA5, vascular mimicry 


\section{INTRODUCTION}

Glioma is a highly aggressive primary brain tumor. The annual incidence of glioma is about 3-6/100,000 people, with a median post-diagnostic survival time of 14.6 months. The combination of radiotherapy and temozolomide (TMZ)-based chemotherapy has become the standard non-operative treatment (1). TMZ is an oral alkylating agent that methylates the guanine at $N^{7}$ and $O^{6}$ and adenine at $O^{3}$ on genomic DNA to form a mismatch during cell replication, resulting in permanent breakage of the DNA chain and cell death, consequently. However, only about $45 \%$ of glioma patients had short-term response to TMZ treatment, while the 5-year survival rate after TMZ treatment was even less than $10 \%$ in glioma patients (2). Previous research works have unearthed multiple TMZ-resistant mechanisms. For example, the $\mathrm{O}^{6}$-methylguanine methyltransferase (MGMT)-mediated DNA repair machine can debug the TMZ-induced genomic mismatch, while epigenetic silencing of MGMT restores sensitivity to TMZ, along with longer overall survival (OS) in patients showing MGMT methylation $(3,4)$. Hitherto, there is no specific remedy for this complicated problem.

By means of multi-omics technology, a hypoxia environment has been proven to take charge of triggering TMZ resistance somehow. It is acknowledged that hypoxia is a key characteristic of glioma. Multiple hypoxia-responsive molecules augmented in glioma are responsible for its invasive and aggressive properties, especially hypoxia-induced factor (HIF)-1 $\alpha$ (5). Treatment with TMZ stressed the hypoxia status by activating HIF- $1 \alpha$ and its multiple target genes (6). The PI3K/AKT-dependent epithelialmesenchymal transition (EMT) process was found to be activated by TMZ, which promoted malignancy as feedback (7). Additionally, stimulation of HIF- $1 \alpha$ can also lead to the acquisition of stem status, denoted by an increased CD133 expression (8). The hypoxia-driven stem-like cell was found to express a high level of MGMT and display stronger chemotherapy resistance in turn (9). If placed under a hyperoxic condition, chemoresistant human glioma cells can be re-sensitized to TMZ (10).

Hypoxia also contributes to multidrug resistance (MDR) in a number of TMZ-resistant glioma patients. Glioma patients who relapsed with TMZ resistance feature faster tumor growth and more abundant tumor vessels. Therefore, anti-angiotherapy was proposed as an ideal option to combat the drug limitation (11). Bevacizumab is a recombinant monoclonal antibody (bevacizumab) approved for the treatment of recurrent glioma, targeting and blocking vascular endothelial growth factor (VEGF), which is a pivotal stimulator of tumor vascularization. However, several clinical trials and laboratory research have proven that current anti-VEGF anti-angiotherapy was poorly effective in TMZ-resistant glioma patients (12). The TMZBevacizumab combined therapy failed to inhibit the tumor or prolong survival in glioma patients, as expected, while the molecular mechanism remains to be explored (13).

In recent years, the phenomenon of vascular mimicry (VM) has been commonly observed and considered to contribute to drug resistance $(14,15)$. In general, HIF- $1 \alpha$ stimulates VEGF to boost the differentiation of pericarcinoma endothelial cells into vascular cells, namely, CD31 ${ }^{+}$vessels (16). Under hypoxia stress, the tumor cells themselves can differentiate into tumor-derived endothelial cells (TDECs) and form luminal structures through self-deformation and remodeling of the extracellular matrix (ECM) (17). CD31 $1^{-}$and positive periodic acid-Schiff (PAS) staining on channels of VM make it different from endothelial cell-derived vessels (18). Red blood cells can be seen in the lumen, keeping oxygen or energy supply, especially when endothelial original vessels have been damaged (19). Certainly, glioma harboring more VM presents with more malignancy and stronger invasive abilities and drug tolerance (17). The existence of VM may be responsible for dysfunction of classical anti-VEGF therapy in cutting off the VM-based oxygen and nutrition supply, thus being inefficient to eliminate the tumor $(20,21)$. Indeed, glioblastoma (GBM) patients with positive VM possess shorter survival times than VM-negative patients, even in patients with MGMT promoter methylation (22). Targeted intervention of tumor cell-derived blood vessels, such as the use of ibrutinib, significantly increased the response rate to chemotherapy in patients with neuroblastoma (23).

The key events of VM formation include ECM remodeling and the connection and translocation between the VM structure and tumor microvessels. These cellular activities were highly involved in the change of cell polarity and EMT, accompanied by morphological changes such as the accelerated formation of invadopodia (24). Maniotis et al. found that VM channels are rich in integrins, laminin, collagen, and heparan sulfate proteoglycan (25). Integrins are a class of cellular adhesion proteins with signal transduction function, which are widespread on the surface of tumor and neovascular cells. So far, the $18 \alpha$ and $9 \beta$ integrin subunits have been identified to dimerize into more than 20 types, such as $\alpha \mathrm{V} \beta 3$ and $\alpha 5 \beta 1$. Integrin- $\alpha 5$ (ITGA5) was reported to impact the invasive nature of many solid tumors by promoting the EMT pathway (26). ITGA5 located on circulating angiogenic cells have been verified to participate in neovascularization, pointing to poorer outcomes in GBM patients (27). In this study, we analyzed public data to prove the value of ITGA5 in predicting responses to TMZ and Bevacizumab and investigated the role of ITGA5 in mediating VM formation in glioma.

\section{MATERIALS AND METHODS}

\section{Public Data Acquisition}

The omics data and clinical information of low-grade glioma (LGG) and GBM samples were downloaded from the Cancer Genome Atlas (TCGA; https://portal.gdc.cancer.gov/) and the Chinese Glioma Genome Atlas (CGGA; http://www.cgga.org.cn/) databases, while those of healthy samples were downloaded from the Genotype-Tissue Expression (GTEx) portal (https://www. gtexportalL.org/) (28). Six hundred and forty-eight (515 LGGs and 133 GBMs) patients with both RNA sequencing and DNA methylation data in TCGA datasets were collected and analyzed. Data of glioma patients showing TMZ/Bevacizumab dual resistance were downloaded from the Gene Expression Omnibus 
(GEO) database (https://www.ncbi.nlm.nih.gov/geo/; GSE79671) (29). Glioma tissue slides with ITGA5 staining were downloaded from The Human Protein Atlas (THPA) database (https://www. proteinatlas.org/). The immunostained level for each slide was evaluated using ImageJ software and compared.

\section{Omics Data Analysis}

For transcriptional sequencing data, differentially expressed genes (DEGs) were analyzed using these data in $\mathrm{R}$ platform by using the "DESeq2" and "ggplot2" packages, with the definition of fold change $>1.5$ and false discovery rate (FDR) $<0.05$. Gene Ontology (GO) analysis was then presented using the DEGs, and the differentially expressed pathways or symptoms were illustrated using gene set enrichment analysis (GSEA) software. The R package "corrplot" was utilized to calculate and exhibit the correlation coefficients among the target genes. The medication and clinical follow-up information of patients were integrated to perform Kaplan-Meier analysis using the R package "survival". The weighted correlation network analysis (WGCNA) was performed using the transcriptome data from TCGA database as the data source (30). The correlations of DEGs were systematically analyzed to calculate the parameter $\beta$ and detect modules. Then, the relationship between the modules and TMZ resistance characteristics was investigated to determine the topranked modules with the strongest connections.

\section{Cell Lines and Cell Culture}

U87MG, the human glioma cell line, was cultured in Dulbecco's modified Eagle's medium (DMEM) supplemented with $10 \%$ fetal bovine serum at $37^{\circ} \mathrm{C}$ under a humidified atmosphere of $5 \% \mathrm{CO}_{2}$.

For the ITGA5-overexpressing cell line, the coding sequence of ITGA5 (NM_002205.5) was cloned in segments and multifragment recombined with the PLVX-puro vector (632164; Clontech, Shiga, Japan). After confirmation of the sequence, the ITGA5-overexpression plasmid was transfected into 293T packaging cells with pSPAX2 and pMD2.G to produce ITGA5overexpressing lentiviral particles. U87MG cells pre-seeded in six-well plates were infected using a medium containing ITGA5overexpressing lentiviral particles supplemented with $10 \mu \mathrm{g} / \mathrm{ml}$ polybrene. At $72 \mathrm{~h}$ after infection, $2 \mu \mathrm{g} / \mathrm{ml}$ puromycin was used to screen cells overexpressing ITGA5, named U87MG-ITGA5.

For the TMZ-resistant GBM cell line, U87MG cells were exposed to the $\mathrm{IC}_{50}$ of TMZ (HY-17364; MedChemExpress, Monmouth Junction, NJ, USA) and then treated continuously with the $\mathrm{IC}_{50}$ of TMZ for 3 months. The TMZ-resistant subclones (U87MG-R) were isolated and maintained in DMEM with a low dose $(100 \mu \mathrm{M})$ of TMZ.

\section{Cytotoxicity Assay}

Cytotoxicity was measured by the sulforhodamine B (SRB) assay (Sigma-Aldrich, St. Louis, MO, USA). The cells were seeded in 96-well plates $(5,000$ cells/well) and cultured in the absence or presence of TMZ $(250,500,1,000,2,000$, and 3,000 $\mu \mathrm{M})$ for $96 \mathrm{~h}$. The cell density was determined by absorbance (optical density, OD) at $490 \mathrm{~nm}$. The percentages of viable cells relative to the controls (cells without previous TMZ treatment) were calculated and plotted. The $\mathrm{IC}_{50}$ values were calculated by derivation of the best-fit line.

\section{Western Blot}

The total protein lysates $(30 \mu \mathrm{g})$ of cells were separated by $10 \%$ SDS-PAGE using electrophoresis and transferred into a PVDF membrane (0.22 $\mu \mathrm{m}$; Millipore, Bedford, MA, USA). After blocking, the membrane was probed with primary antibodies [ITGA5: 10569-1-AP (31); $\beta$-actin: 66009-1-Ig (32)] (both from Proteintech, Wuhan, China) at $1: 1,000$ dilution for $1 \mathrm{~h}$ at $37^{\circ} \mathrm{C}$ and then washed. Horseradish peroxidase (HRP)-conjugated anti-mouse or anti-rabbit secondary antibodies (SA00001-1 and SA00001-2; Proteintech, Wuhan, China) at 1:5,000 dilution were then used for incubation for $1 \mathrm{~h}$. Immunoreactivity signals were amplified by the ECL Plus Western blotting detection system.

\section{PAS Staining}

Cells seeded on slides were fixed with $10 \%$ formalin at room temperature for $15 \mathrm{~min}$ and then washed twice with phosphatebuffered saline (PBS) for PAS staining following the procedure described in Instruction (G1280; Solarbio, Beijing, China). The slides were treated with periodic acid solution for $10 \mathrm{~min}$ and then with Schiff reagent and placed in the dark for $20 \mathrm{~min}$, followed by staining with hematoxylin solution for 1-2 min. Acidic differentiation solutions were added to remove excess background staining. The slides were then dehydrated, cleared, and the images collected using a microscope (Olympus, Tokyo, Japan) and the FCSnap software.

\section{Transcriptional Sequencing of Cells}

Cells were harvested and total RNA was extracted using the Trizol reagent. Beads with oligo(dT) were used to isolate poly (A)-containing mRNA and ncRNAs after total RNA was collected. Sequenced reads were trimmed for adaptor sequence and masked for low-complexity or low-quality sequences, then mapped to the hg19 whole genome using HISAT2. The sequencing experiment was performed by HaploX Genomic Center, and the raw data were deposited in the GEO database (https://www.ncbi.nlm.nih.gov/geo/; PRJNA753670).

\section{Statistical Analysis}

All data were analyzed using IBM SPSS Statistics for Windows software (ver. 23.0; IBM Corp., Armonk, NY, USA). To compare the EMT factor expression levels and WHO tumor grades, we employed the $\chi^{2}$ test or Fisher's exact probability test. The effects of single variables on OS or progression-free survival (PFS) were estimated by univariate analysis. Data are presented as the mean \pm standard deviation (SD), unless otherwise indicated. At least three independent experiments were performed. Unless stated otherwise, the $t$-test was used to compare groups. GraphPad Prism for Windows software (ver. 6.00; GraphPad, La Jolla, CA, USA) was employed to analyze in vitro data, presented as the mean \pm standard error of the mean (SEM). A $p$-value $<0.05$ was considered to reflect statistical significance. 


\section{RESULTS}

We downloaded the transcriptomic data and clinical information of LGG and GBM patients from TCGA database. A total of 167 patients who received TMZ treatment were included, classified into complete response (CR), partial response (PR), stable disease (SD), progressive disease (PD), and recurrent groups according to their disease progression and clinicopathological characteristics after TMZ treatment (Figure 1A). The survival rates varied among each population, while patients with $\mathrm{PD}$ showed the poorest outcome, as expected (Figure 1B). Patients with $\mathrm{PD}$ and recurrent outcome were classified as TMZ-resistant (TMZ-R, $n=59$ ), while patients with CR, PR, and SD were classified as TMZ-non-resistant (TMZ-NR, $n=108$ ). By analyzing the transcriptomic files, 974 DEGs with |fold change| $>1.5$ and FDR $<0.05$ were screened, including 908 upregulated and 66 downregulated genes in the TMZ-R group compared to the TMZ-NR group (Figure 1C). We performed GO analysis
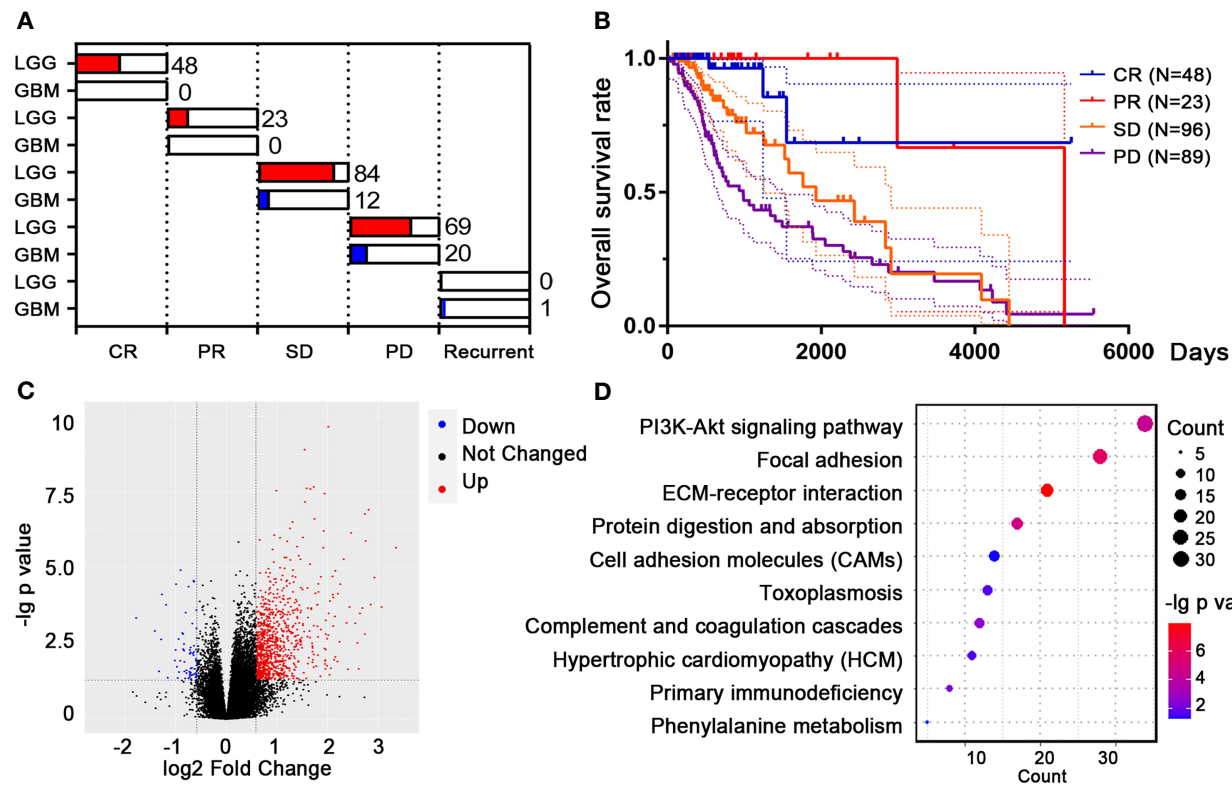

D
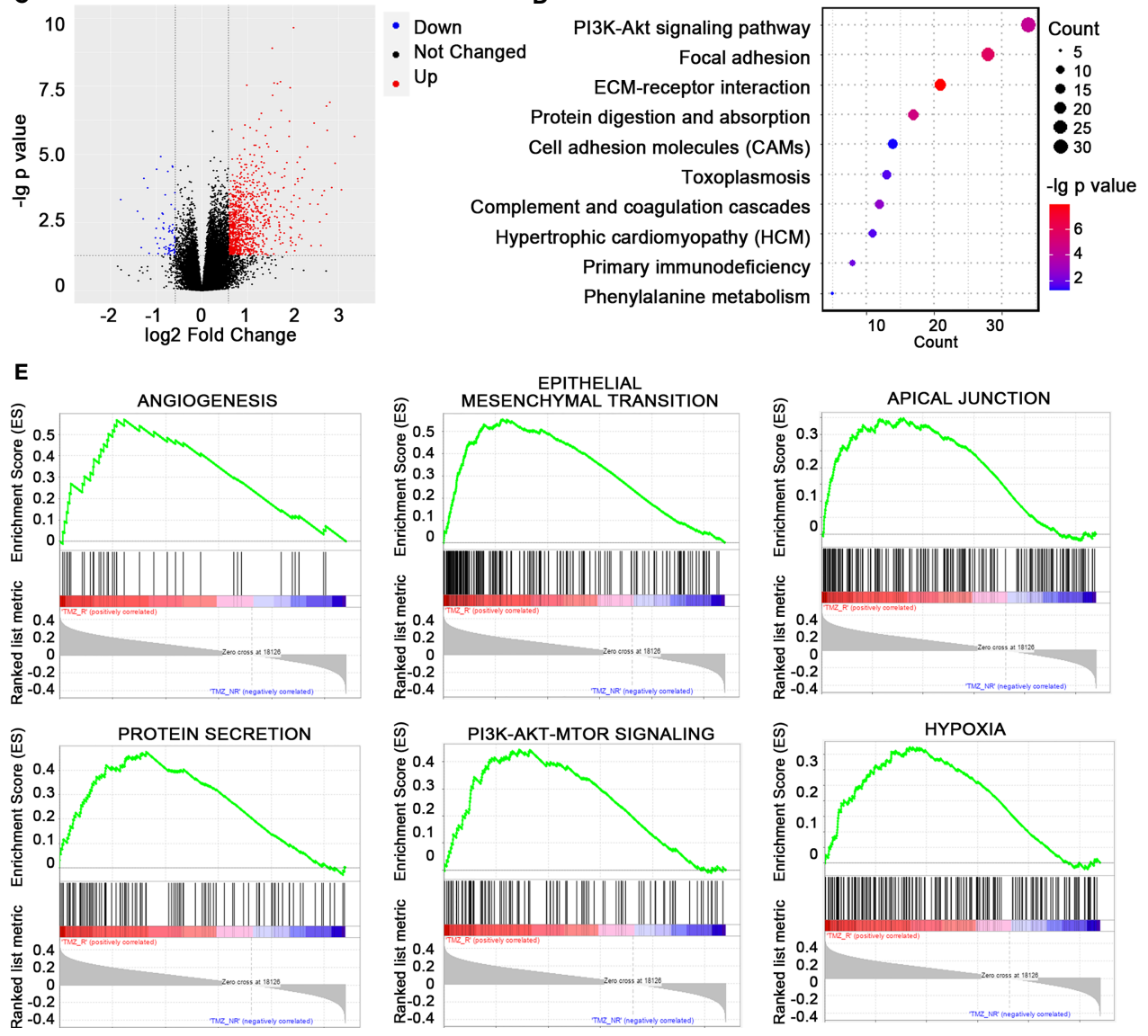

FIGURE 1 | Differentially expressed genes (DEGs) and differential pathway screening between temozolomide-resistant (TMZ-R) and non-resistant (TMZ-NR) glioma patients in The Cancer Genome Atlas (TCGA) database. (A) Based on their responses to TMZ treatment, glioma patients from TCGA database were grouped into TMZ-R [complete response (CR), partial response (PR), stable disease (SD)] and TMZ-NR [progressive disease (PD), and recurrent] groups. (B) The Kaplan-Meier method was used to compute the overall survival of glioma patients with distinct response to TMZ treatment. (C) Volcano plot showing the DEGs between TMZ-R and TMZ-NR glioma patients. The red points refer to upregulated genes, while the blue points refer to downregulated genes in TMZ-R patients. (D) Pathway enrichment of DEGs by Gene Ontology (GO) analysis. (E) The top pathways associated with TMZ-R by gene set enrichment analysis (GSEA). 
using these DEGs and found that biological processes related to cell adhesion and ECM were highly enriched, indicating that the cell morphology was greatly modified (Figure 1D). GSEA was also performed, displaying that clusters of EMT, PI3K/AKT pathway, and angiogenesis process were enriched in the TMZ$\mathrm{R}$ group (Figure 1E).

We presented these DEGs into WGCNA streamline to construct a co-expression genetic network. Seven efficient gene modules were obtained through a one-step network construction method (Figure 2A). Except for the red module, the expressions of the other modules were highly positively correlated with each other, indicating a possible synergy among modules related to TMZ resistance (Figure 2B). The genes of each module, excluding the red module, were extracted for gene function analysis. The enrichment results of the other six modules included the following: ECM components, exosomes, immune response, vascular construction, cell movement, and cell division (Figure 2C). The above results supported the existence of a close association between TMZ resistance and remodeling of cell morphology, as well as the vascular status in glioma.

The above results guided us to explore the potential correlation between TMZ resistance and Bevacizumab resistance. Consequently, we introduced another batch of transcriptome data from the GEO database (GSE79671). The glioma patients in this content were grouped into dual resistance (TMZ/Bev-DR) and non-resistance (TMZ/Bev-NR). A total of 845 upregulated genes and 970 downregulated genes with $\mid$ fold change $\mid>1.5$ and $p<0.05$ were identified in the TMZ/Bev-DR group (Figure 3A). Following GO analysis, we found that the DEGs were responsible for coding membrane proteins, especially centering on transshipment of cellular components, collagen metabolic pathways, among others (Figures 3B-D). The two batches of DEGs from the above two datasets were intersected to obtain the overlapping factors; 83 upregulated genes and 3 downregulated genes were screened (Figure 3E). A broad range of genes related to cell skeleton and structure were included in these upregulated genes, such as ITGA5, ICAM1, and families of annexin (ANXA1 and ANXA2) and collagen (COL4A1,COL5A1, and COL14A1). Considering the expression levels and biological function of these genes, ITGA5, which was both upregulated in the TMZ single-drug resistance and TMZ/Bevacizumab dual-drug resistance groups, was selected for further analysis and functional verification (Figure 3F).

To determine the possible contribution of ITGA5 in drug resistance, we systematically analyzed the transcriptomic data of glioma patients from TCGA and CGGA databases and normal brain samples from the GTEx database. The expression level of ITGA5 was significantly higher in glioma tissues compared to that in normal brain tissue and steadily increased with advancing glioma
A

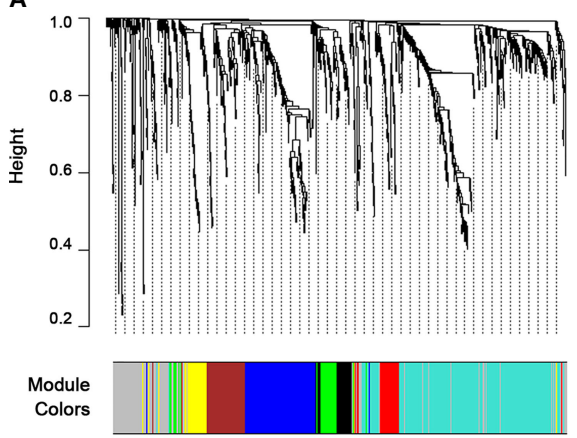

B

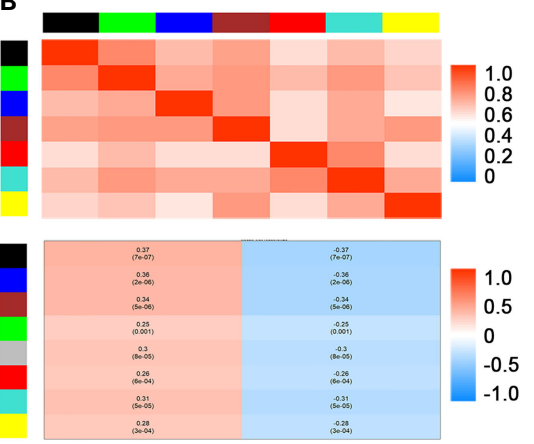

C

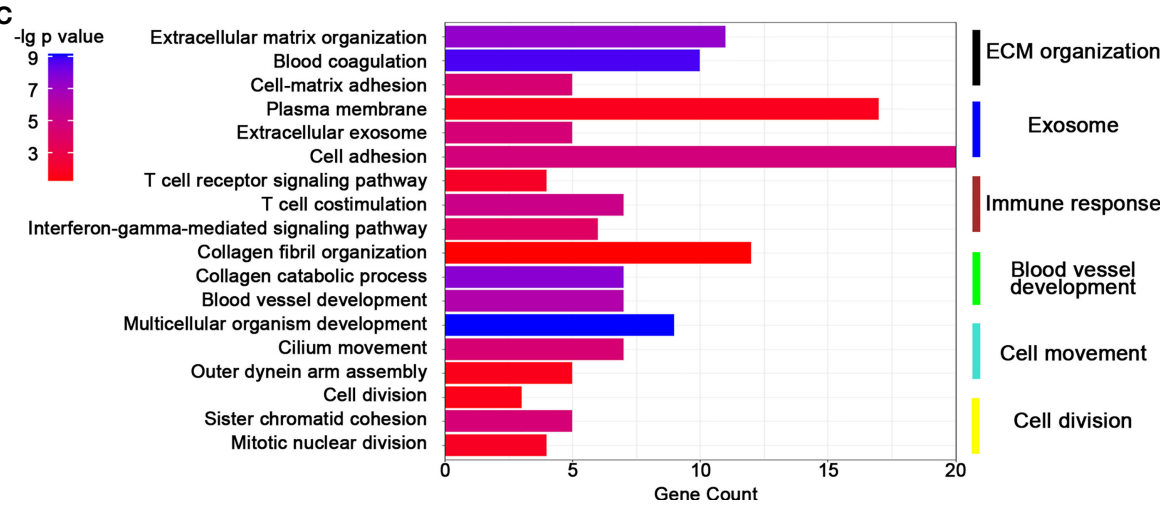

FIGURE 2 | Construction of a weighted co-expression gene network by weighted correlation network analysis (WGCNA). (A) WGCNA dendrogram indicating the expressions of different gene modules in the above glioma samples. (B) Correlations between the gene modules. (C) Heatmap of representative pathways in each gene module. 


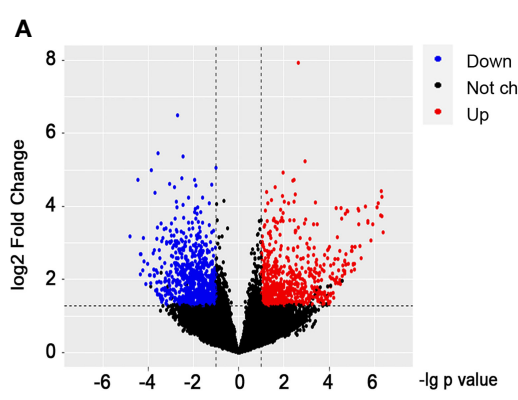

\section{B}

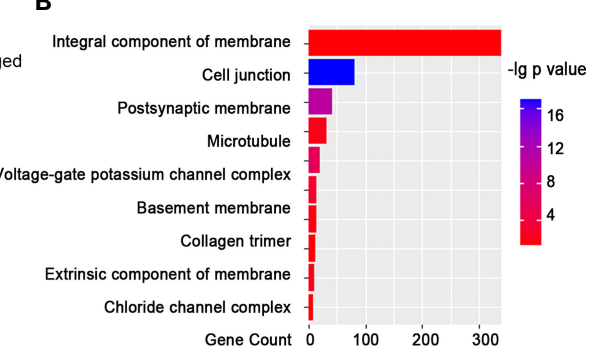

C

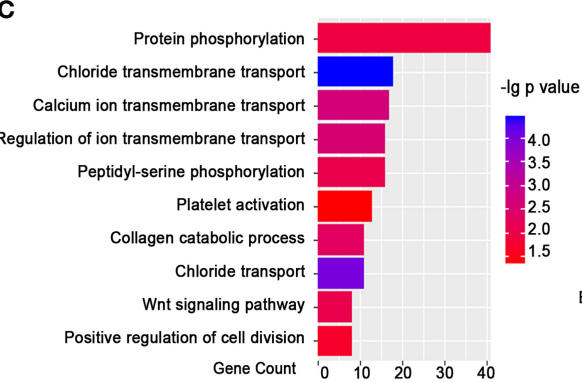

D
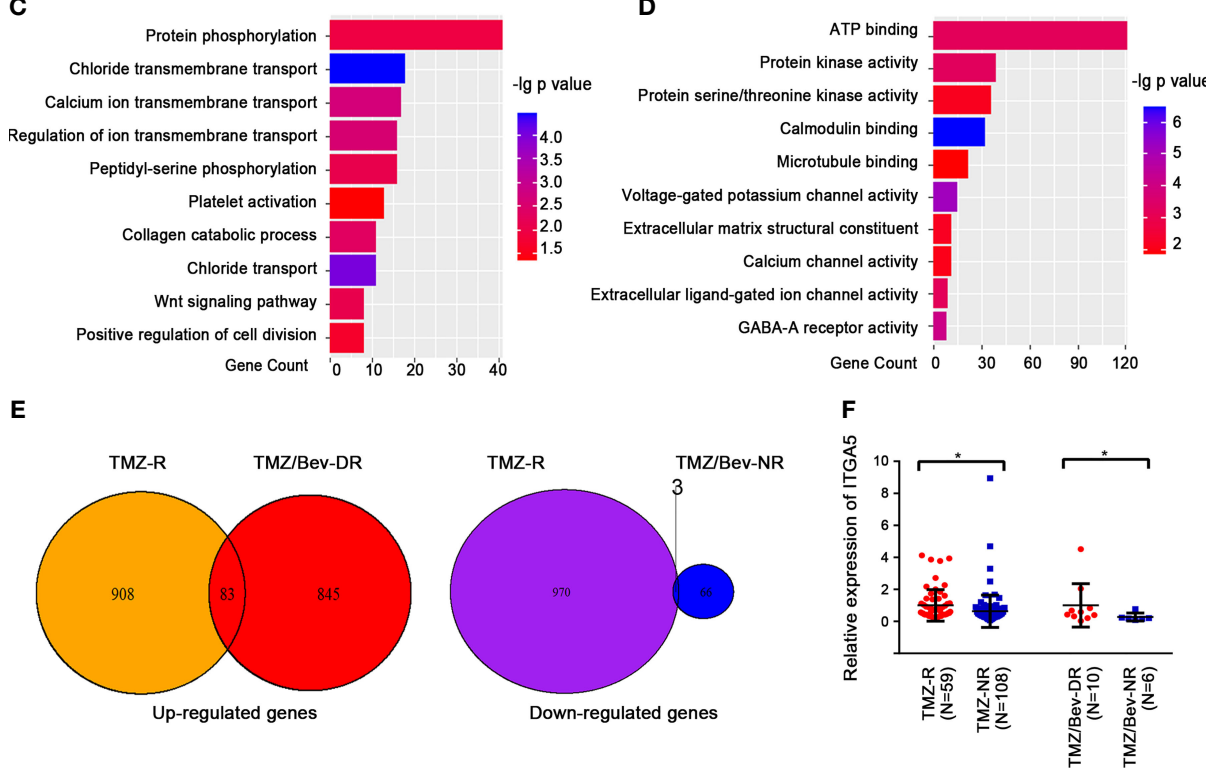

FIGURE 3 | Analysis of candidate genes and related pathways in glioma patients showing dual resistance to temozolomide (TMZ) and Bevacizumab. (A) Volcano plots showing the differentially expressed genes (DEGs) between patients showing dual resistance to TMZ and Bevacizumab (TMZ/Bev-DR) and those not resistant to both drugs (TMZ/Bev-NR). (B-D) Results of the gene function cluster analysis of differential genes, including cellular components (B), biological pathways (C), and molecular functions (D). (E) TMZ/Bev-DR-related differential genes were intersected with the aforementioned TMZ-R-related differential genes according to the changing trend. (F) Comparison of the levels of ITGA5 in glioma patients with different responses to TMZ (and Bevacizumab) treatment $\left({ }^{*} p<0.05\right)$.

grade (Figure 4A). A higher expression level of ITGA5 was associated with poorer outcomes in patients with glioma (TCGA: $\mathrm{HR}=5.564,95 \% \mathrm{CI}=4.203-7.367, p<0.0001$; CGGA: $\mathrm{HR}=2.899$, $95 \% \mathrm{CI}=2.433-3.454, p<0.0001$ ) (Figure 4B). Consistent with this, more abundant ITGA5 protein was observed in high-level gliomas than in low-level gliomas based on the immunohistochemistry staining files from THPA database (Figure 4C). Considering that forming a heterodimer with $\alpha$ - and $\beta$-subunits is necessary for integrin protein function, we calculated the correlation scores between ITGA5 and different $\beta$-subunits and found that the expression of ITGB1 was highly correlated with ITGA5 (Figure 4D). In addition, we also found that ITGA5 was significantly positively correlated with VM-related genes, especially with genes coding collagen and other ECM components (Figures 4E, F), indicating that ITGA5 may increase the degree of malignancy and drug resistance of glioma by affecting VM formation.
Since methylation is pivotal in controlling expression patterns, we explored the DNA methylated landscape of the above patients. As exhibited in Figure 5A, a wider range of methylated genes occurred in the TMZ-NR group, which may explain why more genes were silenced and downregulated. With the thresholds of $\mid$ fold change $\mid>1.2$ and FDR $<0.05$, there were 454 sites with decreased methylation in the TMZ-R group and only one site with increased methylation (Figure 5B). As for the ITGA5 gene, we measured 12 probes targeting different areas on ITGA5 and observed reduced methylation on sites of cg03826594 and cg2379527 in the TMZ-R group, both targeted on the body region of ITGA5 gene (Figure $\mathbf{5 C}$ ). In addition, a negative correlation existed between the methylation scores of these two sites and the gene expression of ITGA5 (Pearson's correlation coefficients of -0.37 and -0.35 , respectively) (Figure 5D). Moreover, the reduced methylation on ITGA5 was associated with poorer outcomes in glioma patients, reinforced by the 

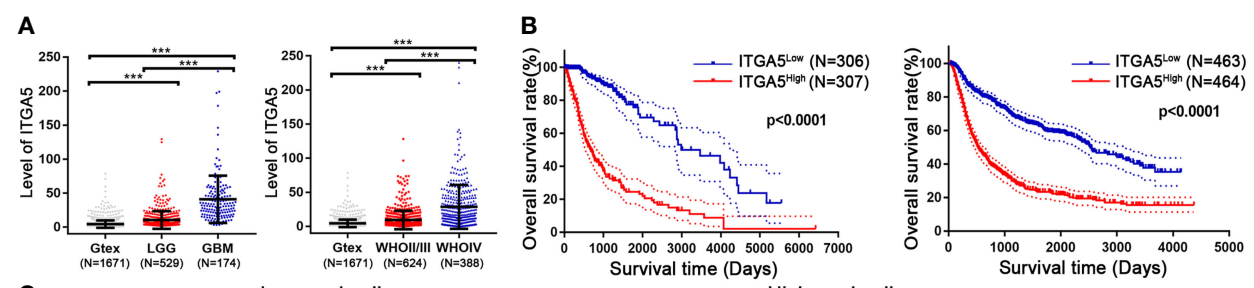

C

Low grade glioma

High grade glioma
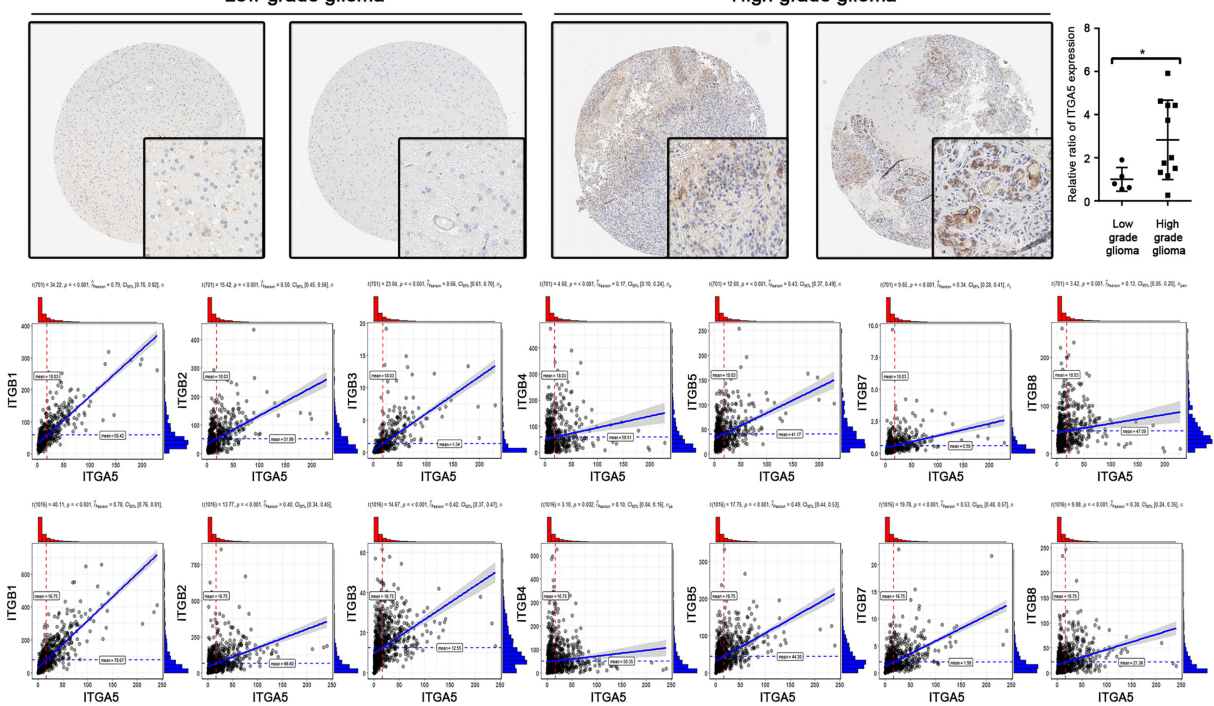

$\mathbf{E}$

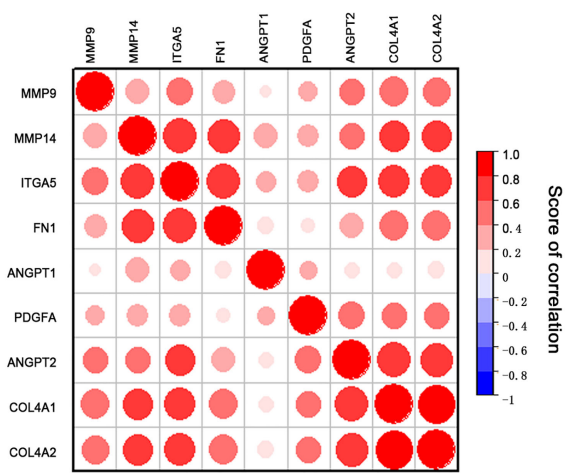

F

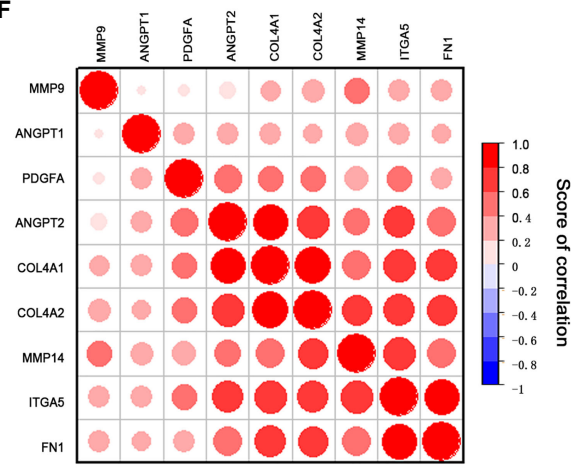

FIGURE 4 | Identification of the prognostic value of ITGA5 in glioma patients from The Cancer Genome Atlas (TCGA) and Chinese Glioma Genome Atlas (CGGA) databases. (A) Transcriptional levels of ITGA5 in glioma patients from TCGA and CGGA. (B) Kaplan-Meier survival curves in glioma patients with high or low expressions of ITGA5. (C) Comparison of the protein levels of ITGA5 in patients' tissues with low- or high-grade glioma from the Human Protein Atlas ( ${ }^{\star} p<0.05$; $\left.{ }^{\star \star \star} p<0.01\right)$. (D) Correlation between ITGA5 and different $\beta$-subunits of integrin using transcriptomic data from TCGA and CGGA. (E, F) Correlation between ITGA5 and the genes responsible for vascular mimicry (VM) using transcriptomic data from TCGA (E) and CGGA (F) databases.

survival analysis (Figure 5E). The above results indicated a striking correlation between reduced methylation and overexpression of ITGA5 in drug-resistant glioma patients.

To confirm the role of ITGA5 in drug resistance, we constructed the TMZ-resistant cell line named U87MG-TMZR, which exhibited a significantly increased resistance to TMZ as verified by the Cell Counting Kit 8 (CCK8) assay (Figure 6A). The results of Western blot showed augmented expression of ITGA5 in TMZR cells compared to that in their parental cells, followed by stronger PAS staining (Figures $\mathbf{6 B}, \mathbf{C}$ ). Then, we constructed and purified ITGA5-overexpressing cells, which was validated by Western blot (Figure 6D). ITGA5-overexpressing cells showed higher resistance to TMZ and a more enriched VM (Figures 6E, F). Moreover, we performed transcriptomic sequencing using these two cell lines. The results showed 619 upregulated genes and 1,537 downregulated genes (|log2 fold change $>1, \mid$ GFOLD $\mid>1$ ). These DEGs were enriched in pathways related to transcription, translation, and cell division (Figure 6G), suggesting that ITGA5 overexpression altered the cell proliferation activity. In addition, the epithelial features were 
A

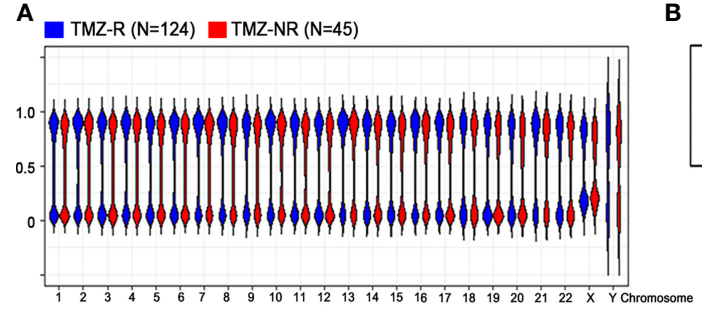

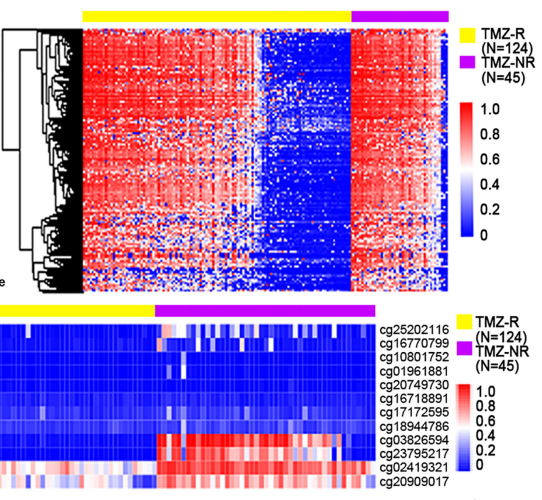

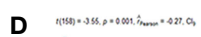

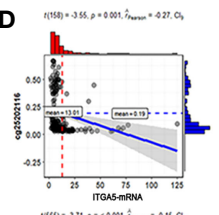

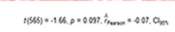
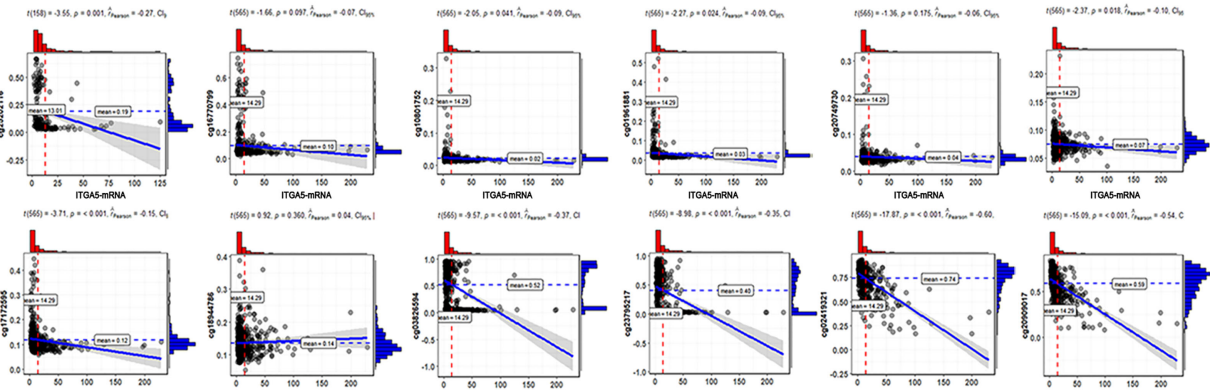

L.
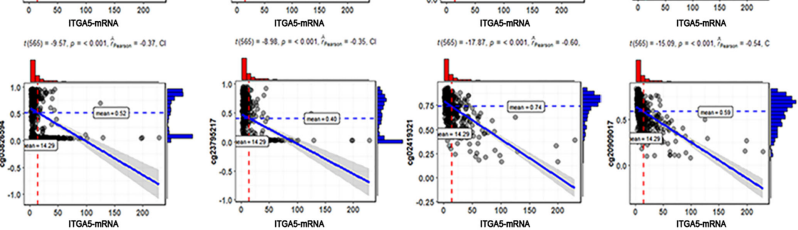

E
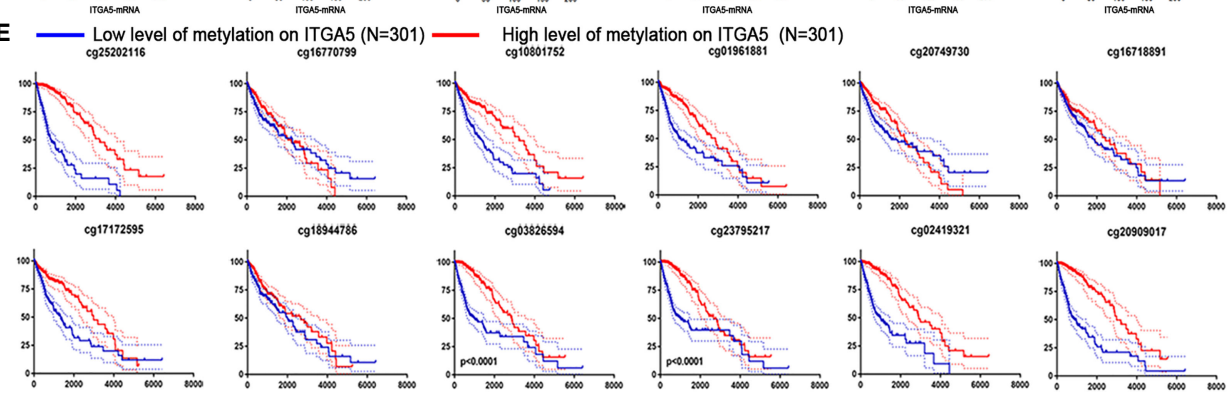

FIGURE 5 | Association between ITGA5 methylation with resistant properties in glioma patients. (A) Methylation landscape in temozolomide-resistant (TMZ-R) and non-resistant (TMZ-NR) glioma patients from The Cancer Genome Atlas (TCGA) database. (B) Differentially methylated sites associated with TMZ resistance in glioma patients. (C) Methylation levels of different sites on the ITGA5 gene. (D) Correlation of the transcriptional level of ITGA5 and the methylation level on its different sites. (E) Kaplan-Meier survival curves in glioma patients grouped by methylation level on the different sites of ITGA5.

decreased with increased mesenchymal genes in ITGA5overexpressing cells, suggesting that ITGA5 promoted the EMT process in glioma cells.

\section{DISCUSSION}

TMZ-based treatment remains a novel therapeutic approach in glioma patients; nonetheless, its therapeutic efficacy remains limited (33). Previous research works have demonstrated that TMZ pressed glioma cells to initiate the EMT process. As a result, TMZ-resistant cells have acquired an EMT-like phenomenon, represented by visible morphological changes (34). Likewise, the change of the morphology and polarity of glioma cells post-therapy changed the vascular environment, making it tolerant to anti-angiotherapy. Numerous clinical pieces of evidence suggest that EMT promotes $\mathrm{VM}$ formation, which is the leading cause of resistance to anti- angiotherapy targeting VEGF in glioma. On the one hand, glioma is a typical vascular-enriched solid tumor with highly heterogeneous vessel structure and sources (35). The rapid tumorigenesis of glioma makes it highly aerobic, especially in the central necrosis region (36). These areas disconnected from the endothelial tissue lack endothelium-dependent vessels, whereas VM channels are often observed nearby (37). Breakage of the existing vessels by chemicals or surgery forces the more robust formation of an intratumor blood supply system, to supply nutrients and oxygen and to remove waste products (38). A more remarkable VM appears in recurrent glioma, which may be induced by chemotherapy-induced hypoxia, to drive tumor resistance and relapse (39). On the other hand, chemical stress endows glioma cells with stemness; a fraction of these GBM stem-like cells can transdifferentiate into vascular smooth musclelike cells to form VM (40). Furthermore, the signal to induce VM can be strengthened under Bevacizumab treatment in an IL-8/ CXCR2-dependent pattern (41). Moreover, VM showed a delayed, 
A
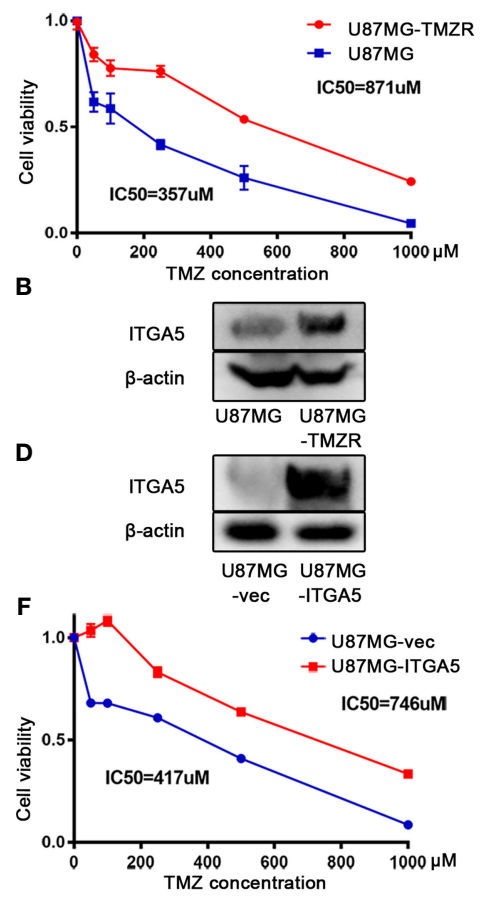

G Down - Not U Up

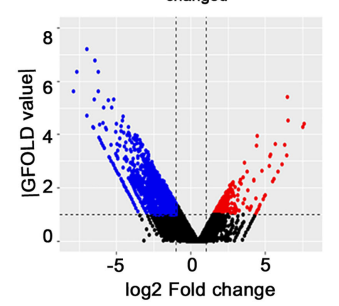

C

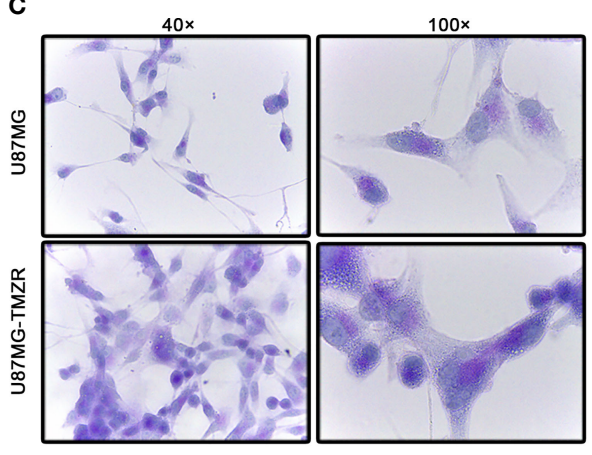

E $40 x$

$100 \times$
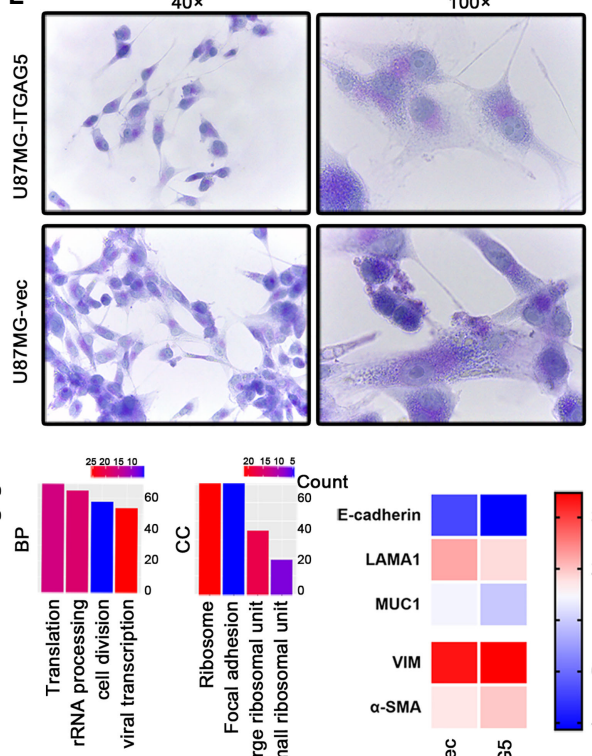
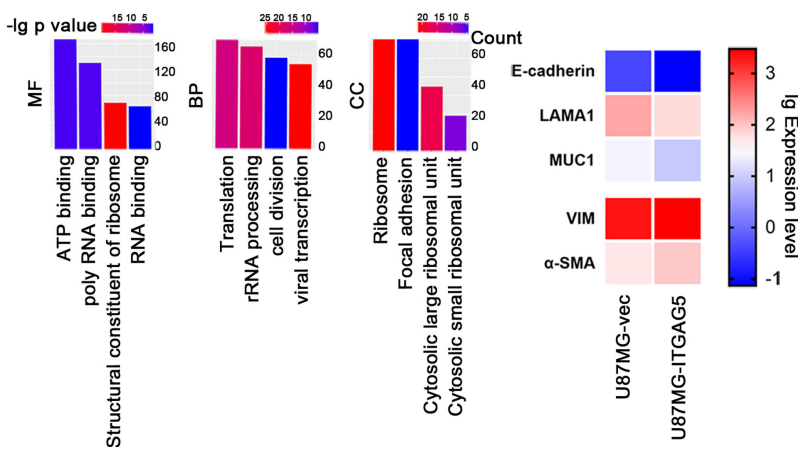

FIGURE 6 | ITGA5 promoted vascular mimicry (VM) formation and temozolomide (TMZ) resistance in glioma cells. (A) Confirmation of a TMZ-resistant (TMZ-R) cell line established using U87MG by Cell Counting Kit 8 (CCK8). (B) Examination of the expression of /TGA5 in U87MG and U87MG-TMZR cells by Western blot. (C) Examination of VM in U87MG and U87MG-TMZR cells by periodic acid-Schiff (PAS) staining. (D) Measurement of ITGA5 expression in ITGA5-overexpressing cells by Western blot. (E) Observation of VM in ITGA5-overexpressing cells by PAS staining. (F) Detection of tolerance to TMZ in ITGA5-overexpressing cells by CCK8. (G) Differentially expressed genes and pathways in ITGA5-overexpressing cells compared to control.

but longer-lasting, vasculogenic activity, contributing to the failure of depriving tumors their blood supply (42). When endothelialderived blood vessels are pharmacologically inhibited, the existence of VM can still support oxygen supply, greatly limiting the therapeutic effect of traditional anti-VEGF drugs (43).

At the beginning of this study, through investigating the DEGs and biological features between TMZ-R and TMZ-NR glioma patients, characteristics related to VM were focused on. In particular, U87MG-TMZR cells exhibited thicker invadopodia than did parental U87MG cells. Invadopodia are actin-rich structures that protrude from the plasma membrane; more robust invadopodia reflect more invasive properties and EMT activity. During radiotherapy or TMZ treatment in GBM cell lines, invadopodia increased along with ECM degradation and remodeling (44). Consistent with previous research, we also noticed that a much stronger activity of invadopodia presented in TMZ-R cells, revealing that the activity of invadopodia bridges the link between EMT and TMZ resistance in gliomas (45). The proteins and signaling pathways parallel in EMT and the generation of invadopodia support a strong association between them (46). The data presented here showed that ITGA5 was greatly involved in these two cellular pathways. Since VM is accompanied by HIF-1 $1 \alpha$, ITGA5 can also be directly induced by HIF-1 $\alpha$ (47). ITGA5 was observed to be localized in invadopodia, while its knockdown reduced the formation of invadopodia in U87MG cells (48). 
Besides with ITGA5, the formation of VM is under the control of multiple ECM components. The major steps in VM formation include: the acquisition of stem-like ability to initiate cell differentiation, ECM remolding to form vascular-like morphology, and physiological connection between the VM channels and endothelial-lined vasculature. The family of integrins is greatly involved in cell adhesion and signal transduction between tumor cells and the ECM. ITGA5 has also been verified to be located on circulating angiogenic cells in GBM, which affects neovascularization (27). After binding with its ligand, signals are transmitted to regulate FAK, $\mathrm{PI} 3 \mathrm{~K}$, and other pathways, affecting tumor growth, invasion, and metastasis (49). Through identifying the "arginine (R)-glycine (G)-aspartate (D)" tripeptide sequence (RGD), integrin combines with ECM skeleton components such as fibrinogen 1 (FN1) and collagen 4 type IV (COL4A1 and COL4A2) to form a cross network in order to maintain the stability of the vascular microstructure (50). This step is assisted by the matrix metalloproteinases (MMP) family by targeting ECM proteins for degradation and releasing related growth factors. Platelet-derived growth factor (PDGF) and angiogenins (ANGPT1 and ANGPT2) can stabilize new blood vessels, while hepatic ligand receptor (EPHA2) signal transduction can regulate cell migration and intercellular adhesion, leading to the maturation of new blood vessels (51). As shown in Figure 4, the expression of ITGA5 is positively correlated with the above ECM factors.

Previous literature has unearthed the existence of multiple DNA repair enzymes contributing to repair TMZ lesions and destroying its antitumor function, which also influenced ITGA5 activity somehow. These mismatch repair (MMR) elements caused genetic alterations during the adaptation to TMZ, endowing glioma cells with new phenomenon in metabolism, proliferation, and immunogenicity. Thereinto, MGMT promoter methylation greatly impacts on MGMT protein expression and TMZ resistance in GBM (52). As exhibited in this article, the DNA methylation level was decreased genome-wide in the TMZ-R group, enabling recovery of the expressions of multiple genes compared to the non-resistant group. Of note is that the methylation level of ITGA5 on two distinct sites (cg03826594 and cg2379527) also showed a close association with ITGA5 expression and TMZ tolerance.

Considering that ITAG5 is a promising therapeutic target, several drugs are undergoing clinical validation. Volociximab, a chimeric immunoglobulin gamma-4 (IgG4) monoclonal antibody binding to ITGA5/B1, demonstrated a favorable phase I safety profile as a single agent (53). MINT1526A (RG-7594) is another fully humanized, high-affinity, and function-blocking anti-human

\section{REFERENCES}

1. Fernandes C, Costa A, Osorio L, Lago RC, Linhares P, Carvalho B, et al. Current Standards of Care in Glioblastoma Therapy. In: De Vleeschouwer S, editor. Glioblastoma. Brisbane (AU): Codon Publications (2017). doi: 10.15586/codon.glioblastoma.2017.ch11

2. Stupp R, Hegi ME, Mason WP, van den Bent MJ, Taphoorn MJB, Janzer RC, et al. Effects of Radiotherapy With Concomitant and Adjuvant Temozolomide Versus Radiotherapy Alone on Survival in Glioblastoma in
ITGA5/B1 IgG1 antibody. MINT1526A inhibits the binding of ITGA5/B1 with FN, thereby blocking ITGA5/B1-mediated endothelial cell adhesion, migration, and sprouting in matrices containing FN. It has also shown good tolerance and preliminary evidence of efficacy when combined with Bevacizumab (54). Cilengitide is an effective inhibitor of ITGAV/B3 and ITGAV/ B5. Treating rats with a combination of bevacizumab and cilengitide significantly restricted tumor invasion than with bevacizumab only, suggesting that inhibiting ITGA5 could replenish the antitumor ability of anti-VEGF agents (55).

In summary, ITGA5-induced VM may promote resistance to TMZ and Bevacizumab by altering glioma vascularization. Besides with briefing the origin and structural characteristics of $\mathrm{VM}$, the elucidation of this mechanism is urgently needed for improving the outcomes of glioma patients and optimizing and developing individualized treatment strategies.

\section{DATA AVAILABILITY STATEMENT}

The datasets presented in this study can be found in online repositories. The names of the repository/repositories and accession number(s) can be found in the article/supplementary material.

\section{AUTHOR CONTRIBUTIONS}

YS, LC, and CX contributed to planning the study. MW, YL, LH, and LX performed the data collection and analysis. YS, HW, and ZL were responsible for the data interpretation. YS and CX drafted the manuscript. AW and LC revised the paper. All authors contributed to the article and approved the submitted version.

\section{FUNDING}

This study was supported by the National Natural Science Foundation of China (no. 81873048) and Sichuan Provincial Science Fund for Distinguished Young Scholars of China (no. 2020JDJQ0065) to CX; the National Natural Science Foundation of China (General Program: no. 81672824; Key Program: no. U20A20380) to LC; Sichuan Science and Technology Program (no. 2021YFH0187) to YS; and the Fundamental Research Funds for the Central Universities (no. ZYGX2020KYQD002) to YS. 
5. Jensen RL. Brain Tumor Hypoxia: Tumorigenesis, Angiogenesis, Imaging, Pseudoprogression, and as a Therapeutic Target. J Neurooncol (2009) 92:31735. doi: 10.1007/s11060-009-9827-2

6. Fisher T, Galanti G, Lavie G, Jacob-Hirsch J, Kventsel I, Zeligson S, et al. Mechanisms Operative in the Antitumor Activity of Temozolomide in Glioblastoma Multiforme. Cancer J (2007) 13:335-44. doi: 10.1097/PPO. 0b013e318157053f

7. Wang P, Zhao L, Gong S, Xiong S, Wang J, Zou D, et al. HIFlalpha/ HIF2alpha-Sox2/Klf4 Promotes the Malignant Progression of Glioblastoma via the EGFR-PI3K/AKT Signalling Pathway With Positive Feedback Under Hypoxia. Cell Death Dis (2021) 12:312. doi: 10.1038/s41419-021-03598-8

8. Lee G, Auffinger B, Guo D, Hasan T, Deheeger M, Tobias AL, et al. Dedifferentiation of Glioma Cells to Glioma Stem-Like Cells By Therapeutic Stress-Induced HIF Signaling in the Recurrent GBM Model. Mol Cancer Ther (2016) 15:3064-76. doi: 10.1158/1535-7163.MCT-15-0675

9. Pistollato F, Abbadi S, Rampazzo E, Persano L, Della Puppa A, Frasson C, et al. Intratumoral Hypoxic Gradient Drives Stem Cells Distribution and MGMT Expression in Glioblastoma. Stem Cells (2010) 28:851-62. doi: $10.1002 /$ stem. 415

10. Sun S, Lee D, Lee NP, Pu JK, Wong ST, Lui WM, et al. Hyperoxia Resensitizes Chemoresistant Human Glioblastoma Cells to Temozolomide. J Neurooncol (2012) 109:467-75. doi: 10.1007/s11060-012-0923-3

11. Gatson NT, Weathers SP, de Groot JF. ReACT Phase II Trial: A Critical Evaluation of the Use of Rindopepimut Plus Bevacizumab to Treat EGFRvIIIPositive Recurrent Glioblastoma. CNS Oncol (2016) 5:11-26. doi: 10.2217/ cns.15.38

12. Paez-Ribes M, Allen E, Hudock J, Takeda T, Okuyama H, Vinals F, et al. Antiangiogenic Therapy Elicits Malignant Progression of Tumors to Increased Local Invasion and Distant Metastasis. Cancer Cell (2009) 15:220-31. doi: 10.1016/j.ccr.2009.01.027

13. Ameratunga M, Pavlakis N, Wheeler H, Grant R, Simes J, Khasraw M. AntiAngiogenic Therapy for High-Grade Glioma: A Meta-Analysis. NeuroOncology (2018) 20:82-3. doi: 10.1093/neuonc/noy148.340

14. Cheng L, Huang Z, Zhou W, Wu Q, Donnola S, Liu JK, et al. Glioblastoma Stem Cells Generate Vascular Pericytes to Support Vessel Function and Tumor Growth. Cell (2013) 153:139-52. doi: 10.1016/j.cell.2013.02.021

15. Baisiwala S, Auffinger B, Caragher SP, Shireman JM, Ahsan R, Lee G, et al. Chemotherapeutic Stress Induces Transdifferentiation of Glioblastoma Cells to Endothelial Cells and Promotes Vascular Mimicry. Stem Cells Int (2019) 2019:6107456. doi: 10.1155/2019/6107456

16. Krock BL, Skuli N, Simon MC. Hypoxia-Induced Angiogenesis: Good and Evil. Genes Cancer (2011) 2:1117-33. doi: 10.1177/1947601911423654

17. Soda Y, Marumoto T, Friedmann-Morvinski D, Soda M, Liu F, Michiue H, et al. Transdifferentiation of Glioblastoma Cells Into Vascular Endothelial Cells. P Natl Acad Sci USA (2011) 108:4274-80. doi: 10.1073/pnas. 1016030108

18. Mei X, Chen YS, Chen FR, Xi SY, Chen ZP. Glioblastoma Stem Cell Differentiation Into Endothelial Cells Evidenced Through Live-Cell Imaging. Neuro Oncol (2017) 19:1109-18. doi: 10.1093/neuonc/nox016

19. Kuang XY, Ren Y, Chen C, Su J, Li HM, Liu SJ, et al. Quantitative Analysis for the Differences in Vasculogenic Activity and Sensitivity to Angiogenic Stimulants Between Human Glioma Cells and Normal Endothelial Cells. Brain Res (2020) 1748:147082. doi: 10.1016/j.brainres.2020.147082

20. Verhoeff JJC, Lavini C, van Linde ME, Stalpers LJA, Majoie C, Reijneveld JC, et al. Bevacizumab and Dose-Intense Temozolomide in Recurrent High-Grade Glioma. Ann Oncol (2010) 21:1723-7. doi: 10.1093/annonc/ mdp591

21. Brandes AA, Stupp R, Hau P, Lacombe D, Gorlia T, Tosoni A, et al. EORTC Study 26041-22041: Phase I/II Study on Concomitant and Adjuvant Temozolomide (TMZ) and Radiotherapy (RT) With PTK787/ZK222584 (PTK/ZK) in Newly Diagnosed Glioblastoma. Eur J Cancer (2010) 46:34854. doi: 10.1016/j.ejca.2009.10.029

22. Mei X, Chen YS, Zhang QP, Chen FR, Xi SY, Long YK, et al. Association Between Glioblastoma Cell-Derived Vessels and Poor Prognosis of the Patients. Cancer Commun (Lond) (2020) 40:211-21. doi: 10.1002/cac2.12026

23. Guerra DAP, Paiva AE, Sena IFG, Azevedo PO, Silva WN, Mintz A, et al. Targeting Glioblastoma-Derived Pericytes Improves Chemotherapeutic Outcome. Angiogenesis (2018) 21:667-75. doi: 10.1007/s10456-018-9621-x
24. Bhattacharya S. Cell Polarity: A Link to Epithelial-Mesenchymal Transition and Vascular Mimicry. Crit Rev Eukaryot Gene Expr (2018) 28:101-5. doi: 10.1615/CritRevEukaryotGeneExpr.2018020775

25. Maniotis AJ, Folberg R, Hess A, Seftor EA, Gardner LM, Pe'er J, et al. Vascular Channel Formation by Human Melanoma Cells In Vivo and In Vitro: Vasculogenic Mimicry. Am J Pathol (1999) 155:739-52. doi: 10.1016/S00029440(10)65173-5

26. Kuninty PR, Bansal R, De Geus SWL, Mardhian DF, Schnittert J, van Baarlen J, et al. ITGA5 Inhibition in Pancreatic Stellate Cells Attenuates Desmoplasia and Potentiates Efficacy of Chemotherapy in Pancreatic Cancer. Sci Adv (2019) 5:eaax2770. doi: 10.1126/sciadv.aax2770

27. Huizer K, Sacchetti A, Swagemakers S, van der Spek PJ, Dik W, Mustafa DA, et al. Circulating Angiogenic Cells in Glioblastoma: Toward Defining Crucial Functional Differences in CAC-Induced Neoplastic Versus Reactive Neovascularization. Neurooncol Adv (2020) 2:vdaa040. doi: 10.1093/noajnl/vdaa040

28. Bao ZS, Chen HM, Yang MY, Zhang CB, Yu K, Ye WL, et al. RNA-Seq of 272 Gliomas Revealed a Novel, Recurrent PTPRZ1-MET Fusion Transcript in Secondary Glioblastomas. Genome Res (2014) 24:1765-73. doi: 10.1101/ gr.165126.113

29. Urup T, Staunstrup LM, Michaelsen SR, Vitting-Seerup K, Bennedbaek M, Toft A, et al. Transcriptional Changes Induced by Bevacizumab Combination Therapy in Responding and Non-Responding Recurrent Glioblastoma Patients. BMC Cancer (2017) 17:278. doi: 10.1186/s12885-017-3251-3

30. Das S, Meher PK, Rai A, Bhar LM, Mandal BN. Statistical Approaches for Gene Selection, Hub Gene Identification and Module Interaction in Gene Co-Expression Network Analysis: An Application to Aluminum Stress in Soybean (Glycine Max L.). PloS One (2017) 12:e0169605. doi: 10.1371/journal.pone.0169605

31. Li J, Chen H, Xu Y, Hu J, Xie FQ, Yang C. Integrin Endocytosis on Elastic Substrates Mediates Mechanosensing. J Biomech (2016) 49:2644-54. doi: 10.1016/j.jbiomech.2016.05.024

32. Lorenzo DN, Bennett V. Cell-Autonomous Adiposity Through Increased Cell Surface GLUT4 Due to Ankyrin-B Deficiency. Proc Natl Acad Sci USA (2017) 114:12743-8. doi: 10.1073/pnas.1708865114

33. Stupp R, Mason WP, van den Bent MJ, Weller M, Fisher B, Taphoorn MJ, et al. Radiotherapy Plus Concomitant and Adjuvant Temozolomide for Glioblastoma. N Engl J Med (2005) 352:987-96. doi: 10.1056/NEJMoa043330

34. Wang J, Zhou F, Li Y, Li Q, Wu Z, Yu L, et al. Cdc20 Overexpression is Involved in Temozolomide-Resistant Glioma Cells With EpithelialMesenchymal Transition. Cell Cycle (2017) 16:2355-65. doi: 10.1080/ 15384101.2017.1388972

35. Jain RK, di Tomaso E, Duda DG, Loeffler JS, Sorensen AG, Batchelor TT. Angiogenesis in Brain Tumours. Nat Rev Neurosci (2007) 8:610-22. doi: $10.1038 / \mathrm{nrn} 2175$

36. Kaur B, Khwaja FW, Severson EA, Matheny SL, Brat DJ, Van Meir EG. Hypoxia and the Hypoxia-Inducible-Factor Pathway in Glioma Growth and Angiogenesis. Neuro Oncol (2005) 7:134-53. doi: 10.1215/S1152851704001115

37. Liu XM, Zhang QP, Mu YG, Zhang XH, Sai K, Pang JC, et al. Clinical Significance of Vasculogenic Mimicry in Human Gliomas. J Neurooncol (2011) 105:173-9. doi: 10.1007/s11060-011-0578-5

38. Jensen RL. Hypoxia in the Tumorigenesis of Gliomas and as a Potential Target for Therapeutic Measures. Neurosurg Focus (2006) 20:E24. doi: 10.3171/ foc.2006.20.4.16

39. Ricci-Vitiani L, Pallini R, Biffoni M, Todaro M, Invernici G, Cenci T, et al. Tumour Vascularization via Endothelial Differentiation of Glioblastoma Stem-Like Cells (Vol 468, Pg 824, 2010). Nature (2011) 469:432-2. doi: 10.1038/nature 09734

40. El Hallani S, Boisselier B, Peglion F, Rousseau A, Colin C, Idbaih A, et al. A New Alternative Mechanism in Glioblastoma Vascularization: Tubular Vasculogenic Mimicry. Brain (2010) 133:973-82. doi: 10.1093/brain/awq044

41. Angara K, Borin TF, Rashid MH, Lebedyeva I, Ara R, Lin PC, et al. CXCR2Expressing Tumor Cells Drive Vascular Mimicry in Antiangiogenic TherapyResistant Glioblastoma. Neoplasia (2018) 20:1070-82. doi: 10.1016/ j.neo.2018.08.011

42. Folkman J. Angiogenesis: An Organizing Principle for Drug Discovery? Nat Rev Drug Discovery (2007) 6:273-86. doi: 10.1038/nrd2115

43. Tian L, Goldstein A, Wang H, Lo HC, Kim IS, Welte T, et al. Mutual Regulation of Tumour Vessel Normalization and Immunostimulatory Reprogramming. Nature (2017) 544:250-+. doi: 10.1038/nature21724 
44. Dinevska M, Gazibegovic N, Morokoff AP, Kaye AH, Drummond KJ, Mantamadiotis T, et al. Inhibition of Radiation and Temozolomide-Induced Glioblastoma Invadopodia Activity Using Ion Channel Drugs. Cancers (Basel) (2020) 12(10):2888. doi: 10.3390/cancers 12102888

45. Ueno H, Tomiyama A, Yamaguchi H, Uekita T, Shirakihara T, Nakashima K, et al. Augmentation of Invadopodia Formation in Temozolomide-Resistant or Adopted Glioma is Regulated by C-Jun Terminal Kinase-Paxillin Axis. Biochem Biophys Res Commun (2015) 468:240-7. doi: 10.1016/j.bbrc.2015.10.122

46. Yilmaz M, Christofori G. EMT, the Cytoskeleton, and Cancer Cell Invasion. Cancer Metastasis Rev (2009) 28:15-33. doi: 10.1007/s10555-008-9169-0

47. Koike T, Kimura N, Miyazaki K, Yabuta T, Kumamoto K, Takenoshita S, et al. Hypoxia Induces Adhesion Molecules on Cancer Cells: A Missing Link Between Warburg Effect and Induction of Selectin-Ligand Carbohydrates. Proc Natl Acad Sci USA (2004) 101:8132-7. doi: 10.1073/pnas.0402088101

48. Mallawaaratchy DM, Buckland ME, McDonald KL, Li CC, Ly L, Sykes EK, et al. Membrane Proteome Analysis of Glioblastoma Cell Invasion. J Neuropathol Exp Neurol (2015) 74:425-41. doi: 10.1097/NEN.0000000000000187

49. Ellert-Miklaszewska A, Poleszak K, Pasierbinska M, Kaminska B. Integrin Signaling in Glioma Pathogenesis: From Biology to Therapy. Int J Mol Sci (2020) 3(10):888. doi: 10.3390/ijms21030888

50. Ruoslahti E. RGD and Other Recognition Sequences for Integrins. Annu Rev Cell Dev Biol (1996) 12:697-715. doi: 10.1146/annurev.cellbio.12.1.697

51. Kim HS, Won YJ, Shim JH, Kim HJ, Kim J, Hong HN, et al. Morphological Characteristics of Vasculogenic Mimicry and Its Correlation With EphA2 Expression in Gastric Adenocarcinoma. Sci Rep-Uk (2019) 26(25):4189-99. doi: 10.1038/s41598-019-40265-7

52. Hegi ME, Liu L, Herman JG, Stupp R, Wick W, Weller M, et al. Correlation of O6-Methylguanine Methyltransferase (MGMT) Promoter Methylation With Clinical Outcomes in Glioblastoma and Clinical Strategies to Modulate MGMT Activity. J Clin Oncol (2008) 26:4189-99. doi: 10.1200/JCO. 2007.11.5964
53. Ricart AD, Tolcher AW, Liu G, Holen K, Schwartz G, Albertini M, et al. Volociximab, a Chimeric Monoclonal Antibody That Specifically Binds Alpha5betal Integrin: A Phase I, Pharmacokinetic, and Biological Correlative Study. Clin Cancer Res (2008) 14:7924-9. doi: 10.1158/10780432.CCR-08-0378

54. Weekes CD, Rosen LS, Capasso A, Wong KM, Ye W, Anderson M, et al. Phase I Study of the Anti-Alpha5betal Monoclonal Antibody MINT1526A With or Without Bevacizumab in Patients With Advanced Solid Tumors. Cancer Chemother Pharmacol (2018) 82:339-51. doi: 10.1007/s00280-018-3622-8

55. Ishida J, Onishi M, Kurozumi K, Ichikawa T, Fujii K, Shimazu Y, et al. Integrin Inhibitor Suppresses Bevacizumab-Induced Glioma Invasion. Transl Oncol (2014) 7:292-302.e1. doi: 10.1016/j.tranon.2014.02.016

Conflict of Interest: The authors declare that the research was conducted in the absence of any commercial or financial relationships that could be construed as a potential conflict of interest.

Publisher's Note: All claims expressed in this article are solely those of the authors and do not necessarily represent those of their affiliated organizations, or those of the publisher, the editors and the reviewers. Any product that may be evaluated in this article, or claim that may be made by its manufacturer, is not guaranteed or endorsed by the publisher.

Copyright $\odot 2021 \mathrm{Shi}, \mathrm{Wu}, \mathrm{Liu}, \mathrm{Hu}, \mathrm{Wu}, \mathrm{Xie}, \mathrm{Liu}, \mathrm{Wu}$, Chen and Xu. This is an openaccess article distributed under the terms of the Creative Commons Attribution License (CC BY). The use, distribution or reproduction in other forums is permitted, provided the original author(s) and the copyright owner(s) are credited and that the original publication in this journal is cited, in accordance with accepted academic practice. No use, distribution or reproduction is permitted which does not comply with these terms. 